\title{
Comparison of the relative entropy of entanglement and negativity
}

\author{
Adam Miranowicz, ${ }^{1,2}$ Satoshi Ishizaka, ${ }^{3,4}$ Bohdan Horst,${ }^{1}$ and Andrzej Grudka ${ }^{1,2,5}$ \\ ${ }^{1}$ Faculty of Physics, Adam Mickiewicz University, 61-614 Poznań, Poland \\ ${ }^{2}$ Institute of Theoretical Physics and Astrophysics, University of Gdańsk, 80-952 Gdańsk, Poland \\ ${ }^{3}$ Nano Electronics Research Laboratories, NEC Corporation, 34 Miyukigaoka, Tsukuba 305-8501, Japan \\ ${ }^{4}$ INQIE, The University of Tokyo, 4-6-1 Komaba, Meguro-ku, Tokyo 153-8505, Japan \\ ${ }^{5}$ National Quantum Information Centre of Gdańsk, 81-824 Sopot, Poland
}

(Dated: October 15, 2018)

\begin{abstract}
It is well known that for two qubits the upper bounds of the relative entropy of entanglement (REE) for a given concurrence as well as the negativity for a given concurrence are reached by pure states. We show that, by contrast, there are two-qubit mixed states for which the REE for some range of a fixed negativity is higher than that for pure states. Moreover, we demonstrate that a mixture of a pure entangled state and pure separable state orthogonal to it is likely to give the maximal REE. By noting that the negativity is a measure of entanglement cost under operations preserving positivity of partial transpose, our results provide an explicit example of operations such that, even though the entanglement cost for an exact preparation is the same, the entanglement of distillation of a mixed state can exceed that of pure states. This means that the entanglement manipulation via a pure state can result in a larger entanglement loss than that via a mixed state.
\end{abstract}

PACS numbers: 03.67.Mn, 03.65.Ud, 42.50.Dv

\section{INTRODUCTION}

In quantifying quantum entanglement of two-qubit mixed states, various measures are commonly applied [1]: the relative entropy of entanglement (REE) [2] a measure of the "distance" (or distinguishability) of an entangled state from the set of disentangled states, the (logarithmic) negativity [3, 4] - a measure of entanglement cost under operations preserving the positivity of partial transpose (PPT) [5], and the concurrence [ [6] - a measure of the entanglement of formation [7].

It can be shown analytically that the upper bounds of the REE for a given concurrence [8] and of the negativity for a given concurrence [9] are reached by pure states. So one could conjecture that pure states have also the highest REE for a given negativity. However, we will demonstrate that there are mixed states exhibiting the REE for a given negativity (in some range) higher than for pure states. Before going into details let us briefly describe the entanglement measures.

\section{ENTANGLEMENT MEASURES}

The relative entropy of entanglement in two-qubit systems according to Vedral et al. can be defined as [2, 8]

$$
E_{R}(\rho)=\min _{\sigma^{\prime} \in \mathcal{D}} S\left(\rho \| \sigma^{\prime}\right)=S(\rho \| \sigma),
$$

where the minimum is taken over the set $\mathcal{D}$ of all separable states $\sigma$, and $S$ is the quantum relative entropy

$$
S(\rho \| \sigma)=\operatorname{Tr}\left(\rho \log _{2} \rho-\rho \log _{2} \sigma\right)
$$

between states $\rho$ and $\sigma$. The REE measures a quasidistance, say $D(\rho \| \sigma)$, of the entangled state $\rho$ from the closest separable state (CSS) $\sigma$. Based on the quantum version of Sanov's theorem, one can also interpret the REE as a measure of statistical distinguishability of $\rho$. The choice of $S(\rho \| \sigma)$ as a candidate for $D(\rho \| \sigma)$ is by no means unique, although this is, to our knowledge, the only proposal that coincides for pure states with the von Neumann entropy of the reduced density operator. Also note that $S(\rho \| \sigma)$ is not symmetric and nor does it satisfy the triangle inequality; thus it is not a true metric.

The negativity $N(\rho)$ for a two-qubit state $\rho$ can be defined by [10, 11, 12]:

$$
N(\rho)=\max \left\{0,-2 \mu_{\min }\right\}
$$

where $\mu_{\min }=\min \operatorname{eig}\left(\rho^{\Gamma}\right)$ is the minimal eigenvalue of the partial transpose, denoted by $\Gamma$, of $\rho$. The negativity is directly related to the Peres-Horodecki criterion [3, 4]. The logarithmic negativity, given by $\log _{2}[N(\rho)+1]$, is a measure of the entanglement cost $E_{C}(\rho)$ under PPT operations [5, 13]. The negativity and logarithmic negativity are monotonically related, reaching unity for Bell states and vanishing for separable states. So for simplicity of our further analysis, we use the negativity instead of the logarithmic negativity.

Another measure of entanglement is the entanglement of formation $E_{F}(\rho)$ [7] or, equivalently for two qubit states, the Wootters concurrence [6] defined as $C(\rho)=$ $\max \left\{0,2 \max _{j} \lambda_{j}-\sum_{j} \lambda_{j}\right\}$, where the $\lambda_{j}$ 's stand for the square roots of the eigenvalues of $\rho\left(\sigma_{y} \otimes \sigma_{y}\right) \rho^{*}\left(\rho_{y} \otimes \sigma_{y}\right)$, and $\sigma_{y}$ is the Pauli spin matrix.

In the last section, we also analyze the entanglement of distillation, $E_{D}(\rho)$ [7], a measure of the entanglement as the fraction of Bell states that can be distilled using the optimal purification protocol. 


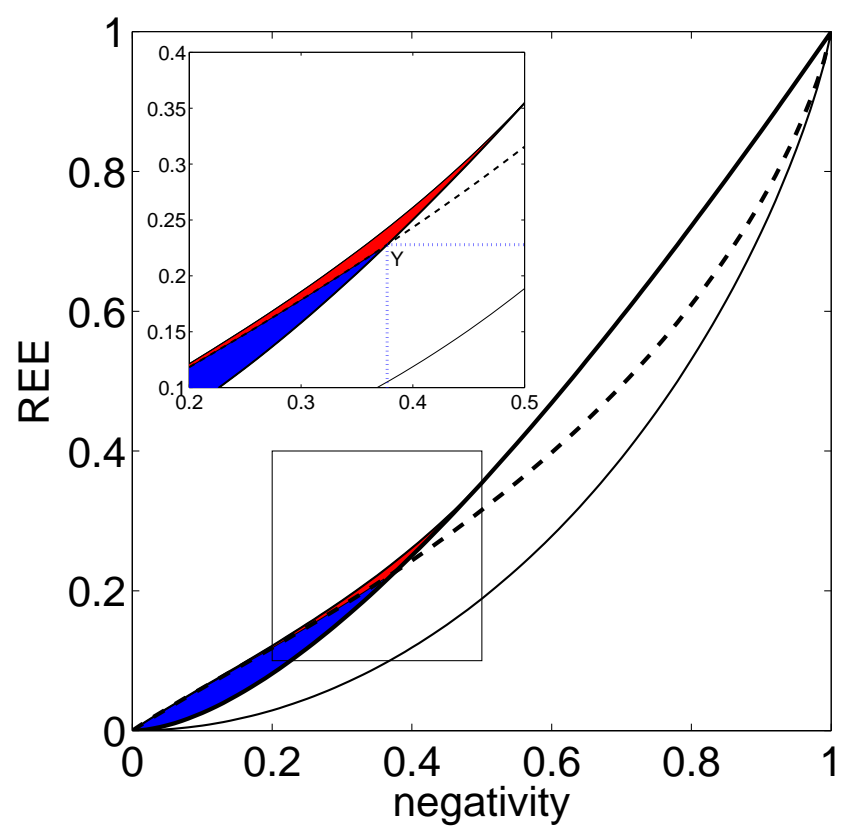

FIG. 1: REE $E_{R}(\rho)$ as a function of negativity $N(\rho)$ for pure states $\rho^{(\mathrm{P})}$ (thick solid curves), the (standard) Horodecki states $\rho^{(\mathrm{H})}$ (dashed curves), the optimal generalized Horodecki states $\rho^{(\mathrm{OGH})}$ (uppermost thin solid curves), and the Bell-diagonal states $\rho^{(\mathrm{BD})}$ (lowest solid curves). Marked regions correspond to states exceeding the pure-state REE: Blue (red) region shows states $\rho^{\left(\mathrm{H}^{\prime}\right)}\left(\rho^{(\mathrm{GH})}\right)$ for which $E_{R}^{\left(\mathrm{H}^{\prime}\right)}>E_{R}^{(\mathrm{P})}\left(E_{R}^{(\mathrm{GH})}>\max \left\{E_{R}^{(\mathrm{P})}, E_{R}^{(\mathrm{H})}\right\}\right)$.

\section{REE WITH FIXED $N$ FOR PURE AND MIXED STATES}

The REE and the entanglement of formation coincide for pure states, but for mixed states the inequality $E_{F}(\rho) \geq E_{R}(\rho)$ holds [8]. As the concurrence is monotonically related to the entanglement of formation for an arbitrary state, the upper bound of the REE for a given concurrence is reached for pure states. On the other hand, as shown by Verstraete et al. [9], the negativity $N(\rho)$ of an arbitrary state can never exceed its concurrence $C(\rho)$. The upper bound of the negativity for a given concurrence, i.e., $C(\rho)=N(\rho)$, is reached for a class of states for which the eigenvector of the partially transposed $\rho$ corresponding to the negative eigenvalue is a Bell state [9, 14, 15]. Pure states and also some mixed states (including Bell-diagonal states) belong to this class. Thus, we see that mixed states cannot give higher values of the REE and negativity for a given concurrence than those for pure states. In the following we will show that the mixed-state REE can exceed the purestate REE for a given negativity.

An arbitrary two-qubit pure state can be changed by local rotations into a state of the form $(0 \leq P \leq 1)$

$$
\left|\psi_{P}\right\rangle=\sqrt{P}|01\rangle+\sqrt{1-P}|10\rangle,
$$

as can be shown by applying the Schmidt decomposi- tion [16]. The negativity of $\left|\psi_{P}\right\rangle$ is simply described by $N\left(\left|\psi_{P}\right\rangle\right)=2 \sqrt{P(1-P)}$, while the REE, being equal to the entanglement of formation, can be given as a function of $N \equiv N\left(\left|\psi_{P}\right\rangle\right)$ as

$$
E_{R}^{(\mathrm{P})}(N) \equiv E_{R}\left(\left|\psi_{P}\right\rangle\right)=H_{2}\left(\frac{1}{2}\left[1+\sqrt{1-N^{2}}\right]\right),
$$

where $H_{2}(x)=-x \log _{2} x-(1-x) \log _{2}(1-x)$ is the binary entropy. Equation (5) corresponds to the well-known Wootters relation between the concurrence and the entanglement of formation [6], since $N\left(\left|\psi_{P}\right\rangle\right)=C\left(\left|\psi_{P}\right\rangle\right)$ and $E_{R}\left(\left|\psi_{P}\right\rangle\right)=E_{F}\left(\left|\psi_{P}\right\rangle\right)$.

In comparison with pure states, let us analyze a mixture of a maximally entangled state, say the "triplet" state $\left|\psi^{+}\right\rangle=(|01\rangle+|10\rangle) / \sqrt{2}$, and a separable state orthogonal to it, say $|00\rangle$, i.e., [17],

$$
\rho^{(\mathrm{H})}=p\left|\psi^{+}\right\rangle\left\langle\psi^{+}|+(1-p)| 00\right\rangle\langle 00|,
$$

where the parameter $p \in\langle 0,1\rangle$. For brevity, we shall refer to (6) as the Horodecki state, although alternatively it could be named after others (see, e.g., [8, 9]). The negativity of the Horodecki state reads as

$$
N\left(\rho^{(\mathrm{H})}\right)=\sqrt{(1-p)^{2}+p^{2}}-(1-p),
$$

while the REE as a function of $N \equiv N\left(\rho^{(\mathrm{H})}\right)$ can be given by Vedral-Plenio's formula [8]

$$
\begin{aligned}
E_{R}^{(\mathrm{H})}(N) & \equiv E_{R}\left(\rho^{(\mathrm{H})}\right)=2 H_{2}(1-p / 2)-H_{2}(p)-p \\
& =(p-2) \log _{2}(1-p / 2)+(1-p) \log _{2}(1-\not \ell \&)
\end{aligned}
$$

where $p=\sqrt{2 N(1+N)}-N$. By comparing the REEs for the Horodecki and pure states we observe that

$$
\begin{array}{ll}
E_{R}^{(\mathrm{H})}(N)>E_{R}^{(\mathrm{P})}(N) & \text { for } 0<N<N_{Y}, \\
E_{R}^{(\mathrm{H})}(N)<E_{R}^{(\mathrm{P})}(N) & \text { for } N_{Y}<N<1,
\end{array}
$$

where $N_{Y}=0.3770 \ldots$ and $E_{R}^{(\mathrm{H})}\left(N_{Y}\right)=E_{R}^{(\mathrm{P})}\left(N_{Y}\right)=$ $0.2279 \ldots$ as shown in the inset plot of Fig. 1. The inequality (9a) can also be shown by expanding (5) and (8) in power series of $N$ close to zero, then one gets $E_{R}^{(\mathrm{H})}(N)=N(1-\sqrt{N / 2}) / \ln 4+\mathcal{O}\left(N^{2}\right)>0$ and negligibly small $E_{R}^{(\mathrm{P})}(N) \sim \mathcal{O}\left(N^{2}\right)$. To show inequality more clearly, we can expand (5) and (8) around $N=1-\epsilon$ close to one, then we have $E_{R}^{(\mathrm{P})}(N)=1-\epsilon / \ln 2+\mathcal{O}\left(\epsilon^{2}\right)$, which is greater than $E_{R}^{(\mathrm{H})}(N)=1-\epsilon(1-\ln \epsilon) / \ln 4+$ $\mathcal{O}\left(\epsilon^{2}\right)$. Thus, a comparison of (5) and (8) demonstrates the main point of this paper: There are mixed states having the REE for a given negativity (in some range) higher than that of pure states.

So far, we have analyzed the Horodecki states, which correspond to the broken curve in Figs. 1 and 2. Now, we will give analytical examples of mixed states more entangled than pure states, which could correspond to any point in the dark region in Fig. 2(a). The desired 

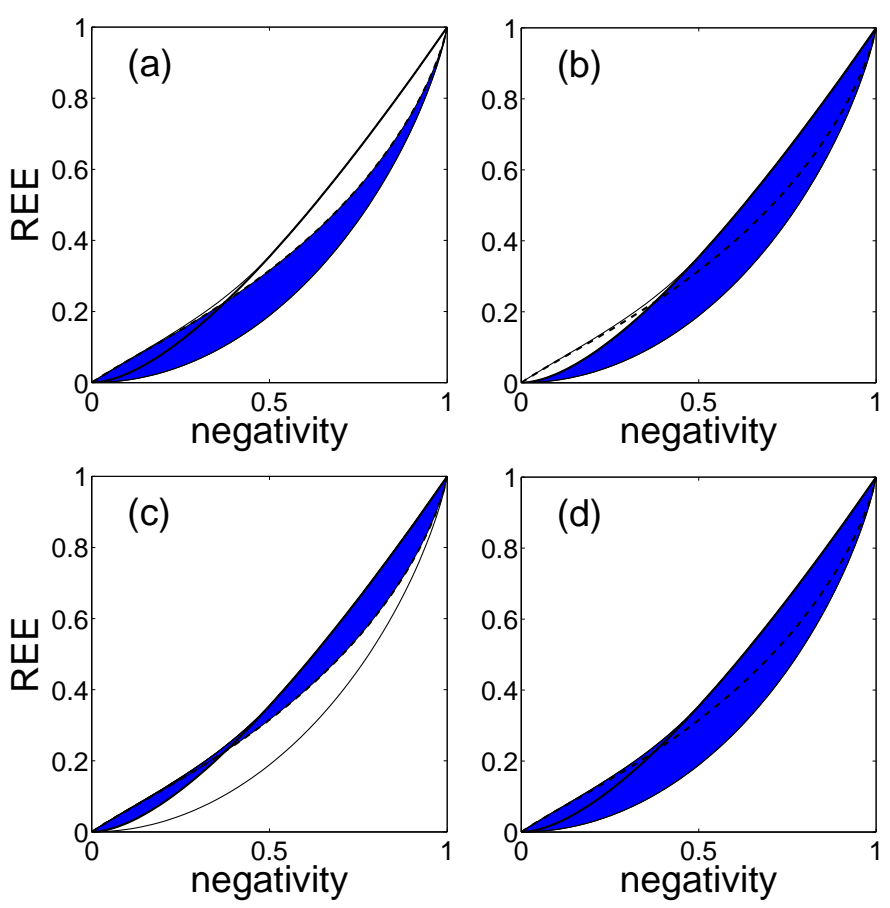

FIG. 2: Ranges of values of the REE with a given negativity for the following states: (a) $\rho^{\left(\mathrm{H}^{\prime}\right)}$ given by (10), (b) $\rho^{\left(\mathrm{P}^{\prime}\right)}$ given by (14), (c) $\rho^{(\mathrm{GH})}$ given by (23), and (d) $\rho^{\left(\mathrm{GH}^{\prime}\right)}$ given by (34). Curves are the same as in Fig. 1.

mixed states can be generated from the Horodecki state $\rho^{(\mathrm{H})}$ by mixing it with the separable state $\sigma^{(\mathrm{H})}$ closest to $\rho^{(\mathrm{H})}$ as follows:

$$
\rho^{\left(\mathrm{H}^{\prime}\right)}(p, N)=(1-x) \rho^{(\mathrm{H})}+x \sigma^{(\mathrm{H})},
$$

defined for $N \in\langle 0,1\rangle$ and $p \in\langle\sqrt{2 N(1+N)}-N, 1\rangle$, where

$$
x=\frac{(N+p)^{2}-2 N(1+N)}{p^{2}(1+N)}
$$

and the corresponding CSS is given by $(q=p / 2)$

$$
\begin{aligned}
\sigma^{(\mathrm{H})}(p)= & q(1-q) \sum_{j, k=0}^{1}(-1)^{j-k}|j, 1-j\rangle\langle k, 1-k| \\
& +(1-q)^{2}|00\rangle\left\langle 00\left|+q^{2}\right| 11\right\rangle\langle 11| .
\end{aligned}
$$

By virtue of the Vedral-Plenio theorem [8], state (12) is the CSS for $\rho^{\left(\mathrm{H}^{\prime}\right)}$ for any $x \in\langle 0,1\rangle$. Thus, we find that the REE for $\rho^{\left(\mathrm{H}^{\prime}\right)}(p, N)$ is given by

$$
\begin{aligned}
& E_{R}\left(\rho^{\left(\mathrm{H}^{\prime}\right)}\right) \equiv E_{R}^{\left(\mathrm{H}^{\prime}\right)}(p, N) \\
= & q^{2} x \log _{2} x+2 q y_{1} \log _{2}\left(\frac{y_{1}}{1-q}\right)+y_{2} \log _{2}\left(\frac{y_{2}}{(1-q)^{2}}\right)
\end{aligned}
$$

where $y_{1}=1-q x, y_{2}=1-2 q+q^{2} x$. The choice of $x$, given by (11), implies that $N$ is just the negativity of
$\rho^{\left(\mathrm{H}^{\prime}\right)}(p, N)$. For $p=p_{0} \equiv \sqrt{2 N(1+N)}-N$, the state (10) goes into the Horodecki state, given by (6). States corresponding to all points in the blue region in Fig. 1 can be generated from $\rho^{\left(\mathrm{H}^{\prime}\right)}(p, N)$ by changing $N$ from 0 to $N_{Y}$ and slightly increasing $p$ from the value of $p_{0}$. By choosing properly $N \in\langle 0,1\rangle$ and $p \in\left\langle p_{0}, 1\right\rangle$, the state $\rho^{\left(\mathrm{H}^{\prime}\right)}(p, N)$ corresponding to any point in the entire dark region in Fig. 2(a) can be generated. It is seen that pure and mixed states having the negativity higher than that $\rho^{(\mathrm{H})}$, which correspond to the white region under the thick solid curve in Fig. 2(a), are not included in the family of states $\rho^{\left(\mathrm{H}^{\prime}\right)}$. By contrast, dark region in Fig. 2(b) corresponds to states that can be obtained from pure states $\left|\psi_{P}\right\rangle$ by mixing them with the separable states $\sigma^{(\mathrm{P})}=P|01\rangle\langle 01|+(1-P)| 10\rangle\langle 10|$ closest to $\rho^{(\mathrm{P})}$. They can be given, in analogy to (10), as

$$
\rho^{\left(\mathrm{P}^{\prime}\right)}(P, N)=(1-x)\left|\psi_{P}\right\rangle\left\langle\psi_{P}\right|+x \sigma^{(\mathrm{P})},
$$

where $x=1-N /[2 \sqrt{P(1-P)}]$ for $N \in\langle 0,1\rangle$ and $P \in$ $\left\langle P_{-}, P_{+}\right\rangle$with $P_{ \pm}=\frac{1}{2}\left(1 \pm \sqrt{1-N^{2}}\right)$. The bounds on $P$ are obtained from the requirement that $\rho^{\left(\mathrm{P}^{\prime}\right)}(P, N)$ should be a positive semidefinite operator. In special cases for $P=P_{ \pm}$, the mixed state $\rho^{\left(\mathrm{P}^{\prime}\right)}\left(P_{ \pm}, N\right)$ becomes the pure state $\rho^{(\mathrm{P})}(N)$. In analogy to the state (10), the Vedral-Plenio theorem guarantees that the CSS for $\rho^{\left(\mathrm{P}^{\prime}\right)}(P, N)$ is the same as for the pure state $\left|\psi_{P}\right\rangle$. Thus, we can calculate the REE for (14) arriving at

$$
\begin{aligned}
& E_{R}\left(\rho^{\left(\mathrm{P}^{\prime}\right)}\right) \equiv E_{R}^{\left(\mathrm{P}^{\prime}\right)}(P, N) \\
= & H_{2}(P)-\frac{z-P y_{-}}{2 P-y_{+}} \log _{2}\left(\frac{y_{-}}{2}\right)-\frac{z-P y_{+}}{2 P-y_{-}} \log _{2}\left(\frac{y_{+}}{2}\right),
\end{aligned}
$$

where $y_{ \pm}=1 \pm \sqrt{1-2 z}$ and $z=2 P(1-P) x(2-x)=$ $2 P(1-P)-N^{2} / 2$. The REE, given by (15), for $P=P_{ \pm}$ goes into (5) as expected. The lower bound of the REEs for both $\rho^{\left(\mathrm{P}^{\prime}\right)}(P, N)$ and $\rho^{\left(\mathrm{H}^{\prime}\right)}(p, N)$ is the same and given by

$$
\begin{aligned}
E_{R}^{(\mathrm{BD})}(N) & \equiv E_{R}^{\left(\mathrm{P}^{\prime}\right)}(1 / 2, N)=E_{R}^{\left(\mathrm{H}^{\prime}\right)}(1, N) \\
& =1-H_{2}\left(\frac{1+N}{2}\right) .
\end{aligned}
$$

With the help of the Vedral et al. results [2], we can conclude that the REE, given by (16), is the same as for a Bell-diagonal state defined by

$$
\rho^{(\mathrm{BD})}=\sum_{i=0}^{3} \lambda_{i}\left|\beta_{i}\right\rangle\left\langle\beta_{i}\right|,
$$

where $\left|\beta_{i}\right\rangle$ are the Bell states, $\sum_{j} \lambda_{j}=1, \max _{j} \lambda_{j}=$ $(1+N) / 2>1 / 2$, and $N$ is the negativity $N\left(\rho^{(\mathrm{BD})}\right)$. Specifically, the states (10) for $p=1$ and (14) for $p=1 / 2$ go into the following Bell-diagonal states

$$
\rho^{\left(\mathrm{H}^{\prime}\right)}(1, N)=\frac{1-N}{4}\left(\left|\beta_{0}\right\rangle\left\langle\beta_{0}|+| \beta_{2}\right\rangle\left\langle\beta_{2}\right|\right)+\frac{1+N}{2}\left|\beta_{3}\right\rangle\left\langle\beta_{3}\right|,
$$




$$
\rho^{\left(\mathrm{P}^{\prime}\right)}(1 / 2, N)=\frac{1+N}{2}\left|\beta_{1}\right\rangle\left\langle\beta_{1}\left|+\frac{1-N}{2}\right| \beta_{3}\right\rangle\left\langle\beta_{3}\right|,
$$

respectively, where the Bell states are given in the following order $\left|\beta_{2 j+k}\right\rangle=\left[|0, k\rangle+(-1)^{j}|1,1-k\rangle\right] / \sqrt{2}$.

The Horodecki state is more entangled than the pure state at most at $N \equiv N^{\prime}=0.1539 \ldots$ for which $\max _{N}\left[E_{R}^{(\mathrm{H})}(N)-E_{R}^{(\mathrm{P})}(N)\right]=0.0391 \ldots$ The question arises about the highest possible difference for an arbitrary mixed state. This problem is strictly related to finding the upper bound of the REE vs negativity.

\section{NUMERICAL SIMULATIONS}

There has been a long-standing open problem of finding a closed analytical formula for the REE for two qubits, which corresponds to finding the $\sigma$ for a given entangled state $\rho$ [19] and it is argued that the analytical solution does not exist [18]. Moreover, there has not yet been an efficient numerical method proposed to calculate the REE for an arbitrary entangled mixed state even in case of two qubits. Analytical formulas for the REE are known only for some special sets of states with high symmetry [2, 8, 18, 20, 21, 22, 23]. Thus, usually, numerical methods for calculating the REE have to be applied [8, 24, 25]. The complexity of the two-qubit problem can be explained by virtue of Caratheodory's theorem, which implies that minimalization of the quantum relative entropy $S(\rho \| \sigma)$ should be performed over 79 real parameters describing decomposed $\sigma$ [8]. Usually [8, 24], gradient-type algorithms are applied to perform the minimalization. Řeháček and Hradil 24] proposed a method resembling a state reconstruction based on the maximum likelihood principle. Doherty et al. 25] designed a hierarchy of more and more complex operational separability criteria for which convex optimization methods (known as semidefinite programs) can be applied efficiently.

Nevertheless, there is a compact-form solution to the inverse problem, which characterizes an entangled state $\rho$ for a given full-rank $\sigma[18,26]$ :

$$
\begin{gathered}
\rho=\sigma-x G(\sigma), \\
G(\sigma)=\sum_{i, j} G_{i j}|i\rangle\left\langle i\left|(|\phi\rangle\langle\phi|)^{\Gamma}\right| j\right\rangle\langle j|,
\end{gathered}
$$

and

$$
G_{i j} \equiv\left\{\begin{array}{cl}
\gamma_{i} & \text { for } \gamma_{i}=\gamma_{j} \\
\frac{\gamma_{i}-\gamma_{j}}{\ln \gamma_{i}-\ln \gamma_{j}} & \text { for } \gamma_{i} \neq \gamma_{j}
\end{array}\right.
$$

and $|\phi\rangle$ is the kernel of $\sigma^{\Gamma}$, while $\mid i>$ and $\gamma_{i}$ are eigenstates and eigenvalues of $\sigma$, respectively. Thus, the REE is given by

$$
E_{R}(\rho)=S(\sigma)-S(\rho)+x \operatorname{tr}\left[(|\phi\rangle\langle\phi|)^{\Gamma} \sigma \log _{2} \sigma\right],
$$

where $S(\cdot)$ is the von Neumann entropy. In the following $x_{\max }$ denotes the largest $x$ in (19), for which $\rho$ is a positive
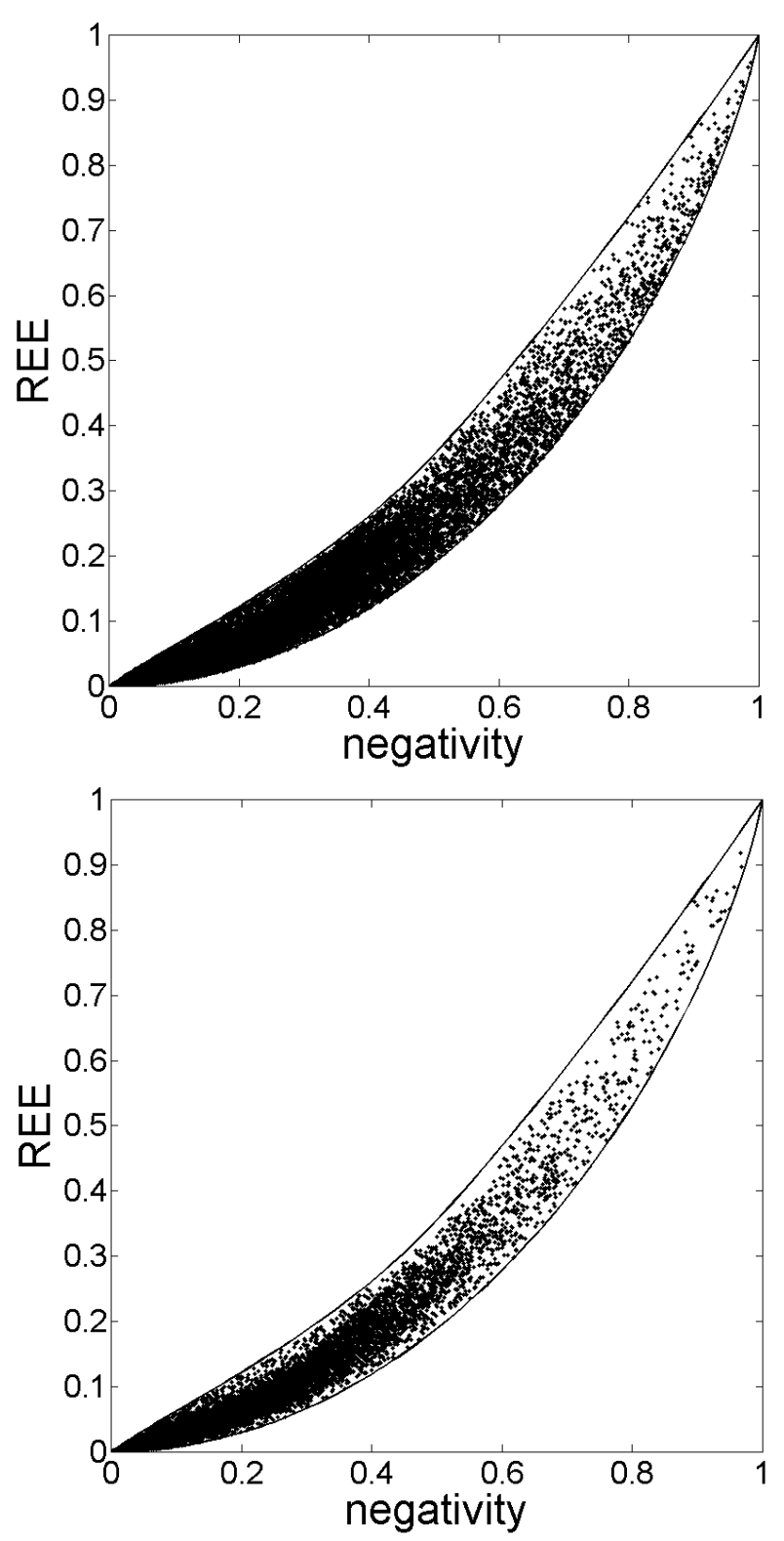

FIG. 3: REE $E_{R}(\rho)$ versus negativity $N(\rho)$ for randomlygenerated states $\rho$ of rank 2 (upper figure) and rank 3 (lower figure). The upper- and lowermost curves correspond to the optimal generalized Horodecki states and Bell-diagonal states, respectively. Random rank-4 states lie in the same range.

operator. The solution can be applied also for lowerrank CSSs in a limiting sequence from a full-rank state by noting that the REE is a continuous function.

We have applied two approaches in our numerical simulations. In the standard approach, the states are chosen at random and their $E_{R}$ and $N$ are calculated numerically using a simplex search method without using numerical or analytic gradients. However, given the fact that no closed formula exists for $E_{R}[18,19]$, it is more logical to choose random states (call them $\sigma$ ) on the boundary of the separability region and generate entangled states $\rho$ 
for which $\sigma$ is the CSS by applying Eqs. (19)-(22). The latter method is faster by three orders of magnitude than the standard one. Figure 3 shows the results of our simulations for random rank- 2 and rank-3 states. For brevity, we have omitted a similar figure for random rank-4 states. The simulations confirm our analytical predictions that the mixed-state REE can exceed the pure-state REE but also indicate lower and upper bounds of the REE vs negativity.

\section{REE FOR THE GENERALIZED HORODECKI STATES}

Our numerical simulations, partially shown in Fig. 3, indicate that the upper bound $E_{R}(N)$ can be given by the rank-2 generalized Horodecki state $\rho^{(\mathrm{GH})}$ defined as follows [18]:

$$
\rho^{(\mathrm{GH})}=p\left|\psi_{P}\right\rangle\left\langle\psi_{P}|+(1-p)| 00\right\rangle\langle 00|,
$$

where $\left|\psi_{P}\right\rangle$ is given by (4) and $p, P \in\langle 0,1\rangle$. In the special case of $P=1 / 2, \rho^{(\mathrm{GH})}$ reduces to the standard Horodecki state, while $\rho^{(\mathrm{GH})}$ for $p=1$ corresponds to a pure state $\left|\psi_{P}\right\rangle$. Note that the negativity $N \equiv N\left(\rho^{(\mathrm{GH})}\right)$ is simply given by:

$$
N=\sqrt{(1-p)^{2}+4 p^{2} P(1-P)}-(1-p) .
$$

By the inversion of this equation,

$$
P=\frac{1}{2 p}\left[p \pm \sqrt{p^{2}-N^{2}-2 N(1-p)}\right]
$$

one can express the state $\rho^{(\mathrm{GH})} \equiv \rho^{(\mathrm{GH})}(p, N)$, given by (23), as a function of negativity $N$ and parameter $p \geq$ $p_{0}(N)=\sqrt{2 N(1+N)}-N$. The state $\rho^{(\mathrm{GH})}$ is a special case of a more general state [18]

$$
\rho^{(\mathrm{Z})} \equiv \rho_{x_{\max }}^{(\mathrm{Z})}=\left(\begin{array}{cccc}
r_{1} & 0 & 0 & 0 \\
0 & r_{2} & y & 0 \\
0 & y & r_{3} & 0 \\
0 & 0 & 0 & 0
\end{array}\right)
$$

for which the CSS is given by:

$$
\sigma^{(\mathrm{Z})}=\left(\begin{array}{cccc}
R_{1} & 0 & 0 & 0 \\
0 & R_{2} & Y & 0 \\
0 & Y & R_{3} & 0 \\
0 & 0 & 0 & R_{4}
\end{array}\right)
$$

where $Y=\sqrt{R_{1} R_{4}}$. Clearly, by assuming $y=\sqrt{r_{2} r_{3}}$, the state $\rho^{(\mathrm{Z})}$ is reduced into the generalized Horodecki state $\rho^{(\mathrm{GH})}$ with $r_{1}=1-p, r_{2}=P p$ and $r_{3}=(1-P) p$. States $\rho^{(\mathrm{Z})}$ and $\sigma^{(\mathrm{Z})}$ are related by the following relations assuming for convenience that $R_{1} \geq R_{4}$ [18]:

$$
r_{2}=R_{2}+\frac{2 R_{4}}{z^{2}}\left(R_{2}^{2}-R_{2} R_{3}+2 Y^{2}\right)+\frac{2 R_{4}}{L z}\left(R_{2}-R_{3}\right)
$$

together with $r_{1}=R_{1}-R_{4}, r_{3}=1-r_{1}-r_{2}$, and $y=$ $-\left[\left(r_{2}-R_{2}\right)\left(R_{2}-R_{3}\right)-2\left(R_{1}+R_{2}\right) R_{4}\right] /(2 Y)$ given in terms of the auxiliary functions $z=\sqrt{\left(R_{2}-R_{3}\right)^{2}+4 Y^{2}}$ and $L=\ln \left(R_{2}+R_{3}-z\right)-\ln \left(R_{2}+R_{3}+z\right)$. Moreover, $x_{\max }=$ $\left(R_{1}+R_{4}\right) / R_{1}$ if the condition $y=\sqrt{r_{2} r_{3}}$ is satisfied for a given choice of $\left\{R_{i}\right\}$.

These equations can easily be inverted for $P=1 / 2$, which leads to the solution given by (12) for the standard Horodecki state $\rho^{(\mathrm{H})}$. By contrast, due to presence of logarithmic functions of nonlinear combinations $\left\{R_{i}\right\}$ in the equations for $\left\{r_{i}\right\}$, it looks impossible to invert the equations in order to express all $\left\{R_{i}\right\}$ in terms of $\left\{r_{i}\right\}$ for the generalized Horodecki state if $P \neq 0, \frac{1}{2}, 1$. Thus, we can only give a formula for the REE for $\rho^{(\mathrm{GH})}$ with $\left\{r_{i}\right\}$ as a function of $\left\{R_{i}\right\}$ :

$E_{R}^{(\mathrm{GH})}=-H_{2}\left(r_{1}\right)-r_{1} \log _{2} R_{1}-f_{-}^{2} \log _{2} \lambda_{-}-f_{+}^{2} \log _{2} \lambda_{+}$,

where $f_{ \pm}=\mathcal{N}_{ \pm}\left[\left(\lambda_{ \pm}-R_{3}\right) \sqrt{r_{2}}+Y \sqrt{r_{3}}\right], \lambda_{ \pm}=\frac{1}{2}\left(R_{2}+\right.$ $\left.R_{3} \pm z\right)$ and $\mathcal{N}_{ \pm}=\left[\left(\lambda_{ \pm}-R_{3}\right)^{2}+Y^{2}\right]^{-1 / 2}$.

In any case, a multivariable numerical procedure for finding the CSS $\sigma^{(\mathrm{GH})}$ can be reduced to a single-variable problem; namely, we can express $R_{i}$ (for $i=2,3,4$ ) in terms of $r_{1}$ and $R_{1}$ as follows:

$$
\begin{aligned}
& R_{2}=\frac{1}{4}\left(1+3 r_{1}+2 r_{2}-4 R_{1}-\sqrt{\delta}\right), \\
& R_{4}=R_{1}-r_{1}, \\
& R_{3}=1-\sum_{i \neq 3} R_{i},
\end{aligned}
$$

where

$$
\begin{aligned}
r_{2,3}= & \frac{1}{2}\left[1-r_{1} \mp \sqrt{\left(1-r_{1}\right)^{2}-N\left(N+2 r_{1}\right)}\right], \\
\delta= & \left(3 r_{1}+1\right)^{2}-4 r_{2} r_{3}-8 R_{1}\left(r_{1}+1\right) \\
& +16 \sqrt{R_{1}\left(R_{1}-r_{1}\right) r_{2} r_{3}} .
\end{aligned}
$$

Thus, to completely determine $\sigma^{(\mathrm{GH})}$ for a given $\rho^{(\mathrm{GH})}$, it is enough to find $R_{1}\left(\geq r_{1}\right)$ by numerically solving the single-variable equation (28) with all the other variables defined above.

A related problem is to find the optimal generalized Horodecki state $\rho^{(\mathrm{OGH})}$, defined as $\rho^{(\mathrm{GH})}$ for a given $N$ and such $p$, denoted by $p_{\text {opt }}(N)$, for which the REE is maximized:

$$
\begin{aligned}
E_{R}^{(\mathrm{OGH})}(N) & \equiv E_{R}\left[\rho^{(\mathrm{OGH})}(N)\right] \\
& \equiv E_{R}\left[\rho^{(\mathrm{GH})}\left(p_{\mathrm{opt}}(N), N\right)\right] \\
& =\max _{p \geq p_{0}(N)} E_{R}\left[\rho^{(\mathrm{GH})}(p(N), N)\right]
\end{aligned}
$$

The parameter $p_{\text {opt }}$ can be found numerically by the procedure described above. On the other hand, we have found a fairly good approximation of $p_{\text {opt }}$ for $0 \leq N \leq$ 0.527 given by

$$
\bar{p}_{\text {opt }}(N)=\frac{1}{3}+\frac{8}{5} N-\frac{7}{11} N^{2}
$$


such that $E_{R}\left[\rho^{(\mathrm{GH})}\left(\bar{p}_{\text {opt }}(N), N\right)\right]$ deviates by the order $10^{-5}$ from the precise value of $E_{R}^{(\mathrm{OGH})}(N)$. We also find that $p_{\text {opt }}$ becomes 1 , so the optimum generalized Horodecki state becomes a pure state $\left|\psi_{P}\right\rangle$ for $N \gtrsim 0.53$. It is worth noting that the precision of our numerical calculations of the REE is $\sim 10^{-10} \div 10^{-8}$, and $\max \left(E_{R}^{(\mathrm{H})}, E_{R}^{(\mathrm{P})}\right)$ is smaller than $E_{R}^{(\mathrm{GH})}$ up to 0.0148 (at $N=0.377$ ), so it can clearly be distinguished from the numerical noise.

The REE for the generalized Horodecki states $\rho^{(\mathrm{GH})}$ as a function of $N$ for arbitrary values of $p$ correspond to the dark region in Fig. 2(c). In analogy with the states $\rho^{\left(\mathrm{H}^{\prime}\right)}$, given by (10), one can also define a class of more general states by mixing $\rho^{(\mathrm{GH})}$ with its $\mathrm{CSS} \sigma^{(\mathrm{GH})}$, given by (12), as follows:

$$
\rho^{\left(\mathrm{GH}^{\prime}\right)}=(1-x) \rho^{(\mathrm{GH})}+x \sigma^{(\mathrm{GH})},
$$

where $x \in\langle 0,1\rangle$. As is seen in Fig. 2(d) in comparison to Figs. 3(a) and 3(b), the REE vs $N$ for $\rho^{\left(\mathrm{GH}^{\prime}\right)}$ covers to whole region of the values for randomly generated states.

We conjecture that for any two-qubit state $\rho$ described by the REE $E_{R}^{(\rho)}(N) \equiv E_{R}(\rho)$ as a function of the negativity $N=N(\rho)$, the following inequalities are satisfied:

$$
E_{R}^{(\mathrm{OGH})}(N) \geq E_{R}^{(\rho)}(N) \geq E_{R}^{(\mathrm{BD})}(N),
$$

which simplify to

$$
E_{R}^{(\mathrm{P})}(N) \geq E_{R}^{(\rho)}(N) \geq E_{R}^{(\mathrm{BD})}(N)
$$

for $N \gtrsim 0.53$, where $E_{R}^{(\mathrm{P})}(N)$ and $E_{R}^{(\mathrm{BD})}(N)$ are given by (5) and (16), while $E_{R}^{(\mathrm{GH})}(N)$ is found numerically by the described method using Eqs. (29)-(31). As a partial analytical support of our conjectures, the extremal conditions for the REE with a fixed $N$ for $\rho^{(\mathrm{GH})}$ and $\rho^{(\mathrm{BD})}$ are examined in the next section. We have also performed a numerical analysis, as discussed in Sec. VI, to provide another support of validity of the conjecture. We have generated altogether a few million random states $\rho$ of a fixed rank $(2,3$, and 4$)$ and calculated the negativity and $\mathrm{REE}$ for each of them.

\section{SOME EXTREMAL CONDITIONS FOR REE WITH FIXED $N$}

In the following, we show analytically that the Belldiagonal states and the generalized Horodecki states, thus also pure states and the standard Horodecki states, satisfy some extremal conditions for the REE with a fixed $N$ implied by a Lagrange-multiplier method. Since negativity for a given state $\rho$ is given by

$$
\begin{aligned}
N(\rho) & =-2 \min _{\left|\psi^{\prime}\right\rangle}\left[\left\langle\psi^{\prime}\left|\rho^{\Gamma}\right| \psi^{\prime}\right\rangle\right] \\
& =-2 \min _{\left|\psi^{\prime}\right\rangle}\left[\operatorname{tr} \rho\left(\left|\psi^{\prime}\right\rangle\left\langle\psi^{\prime}\right|\right)^{\Gamma}\right] \equiv-2\left[\operatorname{tr} \rho(|\psi\rangle\langle\psi|)^{\Gamma}\right],
\end{aligned}
$$

where $|\psi\rangle$ is the optimal state, let us consider the following Lagrange function:

$$
\mathcal{L}=\operatorname{tr} \rho \log _{2} \rho-\operatorname{tr} \rho \log _{2} \sigma+l\left(\operatorname{tr} \rho(|\psi\rangle\langle\psi|)^{\Gamma}+\frac{N}{2}\right),
$$

where $l$ is a Lagrange multiplier. For a small deviation of

$$
\rho \rightarrow \rho+\Delta-(\operatorname{tr} \Delta) \rho,
$$

where $\Delta$ is an arbitrary (but small) operator on the support space of $\rho$ [denoted by $\operatorname{supp}(\rho)$ hereafter], we have

$\mathcal{L} \rightarrow \mathcal{L}+\operatorname{tr} \Delta\left[\log _{2} \rho-\log _{2} \sigma+l(|\psi\rangle\langle\psi|)^{\Gamma}-E_{R}(\rho)+\frac{l}{2} N(\rho)\right]$.

Since $\Delta$ is an arbitrary operator on $\operatorname{supp}(\rho)$, the following extremal condition is obtained:

$P\left[\log _{2} \rho-\log _{2} \sigma+l(|\psi\rangle\langle\psi|)^{\Gamma}-E_{R}(\rho)+\frac{l}{2} N(\rho)\right] P=0$,

where $P$ is the projector to $\operatorname{supp}(\rho)$. Moreover, the extremal condition for $\mathcal{L}$ with respect to $|\psi\rangle$ leads to the extremal condition for negativity, and thus $|\psi\rangle$ must be the eigenstate corresponding to a negative eigenvalue of $\rho^{\Gamma}$. Therefore, it is found that $\rho$, its closest separable state $\sigma$, and the eigenstate $|\psi\rangle$ corresponding to a negative eigenvalue of $\rho^{\Gamma}$ should satisfy Eq. (41).

Now let us consider the case where $\rho$ is a mixed state of rank 2, i.e., $\rho=\lambda_{1}\left|e_{1}\right\rangle\left\langle e_{1}\left|+\lambda_{2}\right| e_{2}\right\rangle\left\langle e_{2}\right|$, where $\left\{\lambda_{i}\right\}$ are nonzero eigenvalues of $\rho$, and $\left|e_{i}\right\rangle$ are corresponding eigenstates. The projector $P$ is then $P=$ $\left|e_{1}\right\rangle\left\langle e_{1}|+| e_{2}\right\rangle\left\langle e_{2}\right|$, and as a result the extremal condition of Eq. (41) becomes

$$
\left\langle e_{1}\left|\log _{2} \sigma\right| e_{2}\right\rangle=l\left\langle e_{1}\left|(|\psi\rangle\langle\psi|)^{\Gamma}\right| e_{2}\right\rangle,
$$

and

$$
\begin{aligned}
\log _{2} \lambda_{1}-\left\langle e_{1}\left|\log _{2} \sigma\right| e_{1}\right\rangle & +l\left\langle e_{1}\left|(|\psi\rangle\langle\psi|)^{\Gamma}\right| e_{1}\right\rangle \\
& =E_{R}(\rho)-\frac{l}{2} N(\rho), \\
\log _{2} \lambda_{2}-\left\langle e_{2}\left|\log _{2} \sigma\right| e_{2}\right\rangle & +l\left\langle e_{2}\left|(|\psi\rangle\langle\psi|)^{\Gamma}\right| e_{2}\right\rangle \\
& =E_{R}(\rho)-\frac{l}{2} N(\rho) .
\end{aligned}
$$

However, Eqs. (43) and (44) are not independent of each other. Indeed, for $\lambda_{1} \neq 0$ and $\lambda_{2} \neq 0$, these equations are equivalent to

$$
\begin{aligned}
\lambda_{1} \log _{2} \lambda_{1}-\lambda_{1}\left\langle e_{1}\left|\log _{2} \sigma\right| e_{1}\right\rangle & +\lambda_{1} l\left\langle e_{1}\left|(|\psi\rangle\langle\psi|)^{\Gamma}\right| e_{1}\right\rangle \\
= & \lambda_{1} E_{R}(\rho)-\lambda_{1} \frac{l}{2} N(\rho), \\
\lambda_{2} \log _{2} \lambda_{2}-\lambda_{2}\left\langle e_{2}\left|\log _{2} \sigma\right| e_{2}\right\rangle & +\lambda_{2} l\left\langle e_{2}\left|(|\psi\rangle\langle\psi|)^{\Gamma}\right| e_{2}\right\rangle \\
= & \lambda_{2} E_{R}(\rho)-\lambda_{2} \frac{l}{2} N(\rho),
\end{aligned}
$$

and it is found that the sum of these equations is automatically satisfied. Therefore, the extremal conditions for rank-2 states are Eqs. (42) and (43) [or Eqs. (42) and (44)]. 


\section{A. Bell-diagonal states}

For the rank-2 Bell-diagonal states,

$$
\left[\rho^{(\mathrm{BD})}, \sigma^{(\mathrm{BD})}\right]=\left[\rho^{(\mathrm{BD})},(|\psi\rangle\langle\psi|)^{\Gamma}\right]=0,
$$

and hence $\left\langle e_{1}\left|\log _{2} \sigma\right| e_{2}\right\rangle=\left\langle e_{1}\left|(|\psi\rangle\langle\psi|)^{\Gamma}\right| e_{2}\right\rangle=0$ holds again. Equation (42) is then satisfied for any $l$, and the extremal conditions are satisfied.

\section{B. Horodecki states}

For the standard Horodecki state, defined by (6), with its CSS given by (12), we have:

$$
\begin{gathered}
\left(\rho^{(\mathrm{H})}\right)^{\Gamma}=q\left(\left|\psi^{+}\right\rangle\left\langle\psi^{+}|+| \psi^{-}\right\rangle\left\langle\psi^{-}|+| 00\right\rangle\langle 11|\right. \\
+|11\rangle\langle 00|)+(1-p)|00\rangle\langle 00|,
\end{gathered}
$$

and

$$
\begin{aligned}
|\psi\rangle= & \sqrt{s_{-}}|00\rangle-\sqrt{s_{+}}|11\rangle, \\
(|\psi\rangle\langle\psi|)^{\Gamma}= & s_{-}|00\rangle\left\langle 00\left|+s_{+}\right| 11\right\rangle\langle 11| \\
& +\frac{t p}{2}\left(\left|\psi^{-}\right\rangle\left\langle\psi^{-}|-| \psi^{+}\right\rangle\left\langle\psi^{+}\right|\right),
\end{aligned}
$$

where $\left|\psi^{ \pm}\right\rangle=(|01\rangle \pm|10\rangle) / \sqrt{2}, s_{ \pm}=[1 \pm t(1-p)] / 2$ and $t=1 / \sqrt{2 p^{2}-2 p+1}$.

Since $\left\langle e_{1}\left|\log _{2} \sigma\right| e_{2}\right\rangle=\left\langle e_{1}\left|(|\psi\rangle\langle\psi|)^{\Gamma}\right| e_{2}\right\rangle=0$, Eq. (42) is satisfied for any $l$. Since there is only one relation of Eq. (43) for $l$, the extremal conditions are necessarily satisfied.

\section{Generalized Horodecki states}

Here, we show that the generalized Horodecki states are extremal. The point is that only two extremal conditions should be satisfied for the states: (i) given by (42) and (ii) given by either of Eqs. (43)-(46) or, e.g., the difference of Eqs. (45) and (46). Condition (ii) is a linear function of $l$, so it can easily be solved for $l$. The question is whether the found $l$ also satisfies condition (i) or whether left- and right-hand sides (LHS and RHS) of (i) are equal to zero. In the following we show that the latter case is satisfied for the generalized Horodecki states $\rho^{(\mathrm{GH})}$, and thus also for the optimal states $\rho^{(\mathrm{OGH})}$, the standard Horodecki states $\rho^{(\mathrm{H})}$, and pure states $\rho^{(\mathrm{P})}$.

For simplicity, we use the notation of Eqs. (26) and (27) with the condition $y^{2}=r_{2} r_{3}$, which guarantees that $\rho^{(\mathrm{Z})}$ and $\sigma^{(\mathrm{Z})}$ become $\rho^{(\mathrm{GH})}$ and $\sigma^{(\mathrm{GH})}$, respectively. One finds that

$$
\begin{aligned}
|\psi\rangle= & \mathcal{N}(-g|00\rangle+2 y|11\rangle), \\
(|\psi\rangle\langle\psi|)^{\Gamma}= & \mathcal{N}^{2}\left[g^{2}|00\rangle\left\langle 00\left|+4 y^{2}\right| 11\right\rangle\langle 11|\right. \\
& \quad-2 g y(|01\rangle\langle 10|+| 10\rangle\langle 01|)],
\end{aligned}
$$

where $g=\sqrt{r_{1}^{2}+4 y^{2}}-r_{1}$ and $\mathcal{N}=1 / \sqrt{g^{2}+4 y^{2}}$. On the other hand, $\log _{2} \sigma^{(\mathrm{GH})}$ can be calculated through the eigenvalue decomposition

$$
\begin{aligned}
\sigma^{(\mathrm{GH})}= & R_{1}|00\rangle\left\langle 00\left|+R_{4}\right| 11\right\rangle\langle 11| \\
& +\lambda_{+}\left|\lambda_{+}\right\rangle\left\langle\lambda_{+}\left|+\lambda_{-}\right| \lambda_{-}\right\rangle\left\langle\lambda_{-}\right|,
\end{aligned}
$$

where

$$
\begin{aligned}
\lambda_{ \pm} & =\frac{1}{2}\left[R_{2}+R_{3} \pm \sqrt{\left(R_{2}-R_{3}\right)^{2}+4 Y^{2}}\right], \\
\left|\lambda_{ \pm}\right\rangle & =\mathcal{N}_{ \pm}\left[\left(\lambda_{ \pm}-R_{3}\right)|01\rangle+Y|10\rangle\right],
\end{aligned}
$$

with $\mathcal{N}_{ \pm}=\left[\left(\lambda_{ \pm}-R_{3}\right)^{2}+Y^{2}\right]^{-1 / 2}$. Moreover, for the nonzero eigenvalues, the eigenvectors of $\rho^{(\mathrm{GH})}$ are found to be $\left|e_{1}\right\rangle=|00\rangle$ and $\left|e_{2}\right\rangle=\left(1 / \sqrt{y^{2}+r_{3}^{2}}\right)\left(y|01\rangle+r_{3}|10\rangle\right)$. Thus, it is seen that both LHS and RHS of condition (42) are equal to zero. The second condition is satisfied by choosing

$$
l=\frac{2 f\left(E_{R}^{(\mathrm{GH})}-\log _{2} r_{1}+\log _{2} R_{1}\right)}{(f+1)\left(f-r_{1}\right)},
$$

where $f=\sqrt{r_{1}^{2}+4 y^{2}}$. In any case, even without knowing explicitly $E_{R}^{(\mathrm{GH})}$ and $\left\{R_{i}\right\}$ in terms of $\left\{r_{i}\right\}$, we have showed that the generalized Horodecki states satisfy the extremal conditions.

\section{CONCLUSIONS}

In conclusion, we have demonstrated that there are mixed states that have the REE in some range of a fixed negativity higher than the pure-state REE for the same negativity. This is somewhat surprising, since mixed states can neither exhibit the REE for a given concurrence nor negativity for a given concurrence higher than those for pure states. By applying Lagrange multipliers, we have also shown that the Bell-diagonal states, pure states, but also the so-called generalized Horodecki states, which are mixtures of a pure entangled state and pure separable state orthogonal to it, satisfy some extremal conditions for the REE with a fixed negativity.

Our findings implicitly show another fact. For a given negativity, the entanglement of distillation (ED) $E_{D}$ of the Horodecki state $\rho^{(\mathrm{H})}$ can be larger than that of a pure state $\rho^{(\mathrm{P})}$. For example, $N=0.1$ and $p=0.37$, one gets $E_{D}\left(\rho^{(\mathrm{P})}\right)=E_{R}\left(\rho^{(\mathrm{P})}\right)=0.025$ and $E_{D}\left(\rho^{(\mathrm{H})}\right)>0.034$. Here, the lower bound of the ED is given by $p^{2} / 4$ (via the direct method shown in [7]). This lower bound can be slightly improved by a method discussed in Ref. 27]. The point is that the logarithmic negativity is equal to a PPT entanglement cost for an exact preparation, the REE is equal to a PPT distillable entanglement for pure states, and the ED is a lower bound of a PPT distillable entanglement. So our findings provide an explicit example of PPT operations where, even though the entanglement cost for an exact preparation is the same, the 
ED of a mixed state can exceed that of pure states. In other words, the entanglement manipulation via a pure state can result in a larger entanglement loss than that via a mixed state.

Acknowledgments. We thank Ryszard Horodecki,
Paweł Horodecki, Michał Horodecki, Zdeněk Hradil, Frank Verstraete, Shashank Virmani and Karol Życzkowski for their pertinent comments. We also appreciate useful suggestions of an anonymous referee. The research was conducted within the LFPPI network.
[1] R. Horodecki, P. Horodecki, M. Horodecki and K. Horodecki, e-print arXiv:quant-ph/0702225 (to be published).

[2] V. Vedral, M. B. Plenio, M. A. Rippin, and P. L. Knight, Phys. Rev. Lett. 78, 2275 (1997).

[3] A. Peres, Phys. Rev. Lett. 77, 1413 (1996).

[4] M. Horodecki, P. Horodecki, and R. Horodecki, Phys. Lett. A 223, 1 (1996).

[5] K. Audenaert, M. B. Plenio, and J. Eisert, Phys. Rev. Lett. 90, 027901 (2003).

[6] W. K. Wootters, Phys. Rev. Lett. 80, 2245 (1998).

[7] C. H. Bennett, D. P. DiVincenzo, J. A. Smolin, and W. K. Wootters, Phys. Rev. A 54, 3824 (1996).

[8] V. Vedral and M. B.Plenio, Phys. Rev. A 57, 1619 (1998).

[9] F. Verstraete, K. M. R. Audenaert, J. Dehaene, and B. De Moor, J. Phys. A 34, 10327 (2001).

[10] K. Życzkowski, P. Horodecki, A. Sanpera, and M. Lewenstein, Phys. Rev. A 58, 883 (1998).

[11] J. Eisert and M. Plenio, J. Mod. Opt. 46, 145 (1999).

[12] G. Vidal and R. F. Werner, Phys. Rev. A 65, 032314 (2002).

[13] S. Ishizaka, Phys. Rev. A 69, 020301(R) (2004).

[14] A. Miranowicz and A. Grudka, Phys. Rev. A 70, 032326 (2004),

[15] A. Miranowicz and A. Grudka, J. Opt. B: Quantum Semiclass. Opt. 6, 542 (2004).

[16] A. Peres, Quantum Theory: Concepts and Methods
(Kluwer Academic, Dordrecht, Holland, 1993).

[17] M. Horodecki, P. Horodecki, and R. Horodecki, in Quantum Information: An Introduction to Basic Theoretical Concepts and Experiments, edited by G. Alber et al. (Springer, Berlin, 2001), p. 151.

[18] A. Miranowicz and S. Ishizaka, Phys. Rev. A 78, 032310 (2008).

[19] J. Eisert, Problem 8: Qubit formula for relative entropy of entanglement, in: Some Open Problems in Quantum Information Theory, eds. O. Krueger, R.F. Werner, eprint arXiv:quant-ph/0504166 1 .

[20] V. Vedral, M. B. Plenio, K. Jacobs, and P. L. Knight, Phys. Rev. A 56, 4452 (1997).

[21] K. G. H. Vollbrecht and R. F. Werner, Phys. Rev. A 64, 062307 (2001).

[22] K. M. R. Audenaert, J. Eisert, E. Jané, M. B. Plenio, S. Virmani, and B. De Moor, Phys. Rev. Lett. 87, 217902 (2001).

[23] K. M. R. Audenaert, B. De Moor, K. G. H. Vollbrecht, and R. F. Werner, Phys. Rev. A 66, 032310 (2002).

[24] J. Řeháček and Z. Hradil, Phys. Rev. Lett. 90, 127904 (2003).

[25] A. C. Doherty, P. A. Parrilo, and F. M. Spedalieri, Phys. Rev. Lett. 88, 187904 (2002).

[26] S. Ishizaka, Phys. Rev. A 67, 060301(R) (2003).

[27] S. Ishizaka (unpublished). 